(Se) former pour enseigner le français à ceux qui ne le parlent pas nativement -

\title{
Le rôle des écoles dans la mission des Églises anglicanes et protestantes du Sud : héritages, mutations, perspectives
}

Jean-François Zorn

\section{(2) OpenEdition}

\section{Édition électronique}

URL : https://journals.openedition.org/dhfles/4259

DOI : $10.4000 /$ dhfles.4259

ISSN : 2221-4038

\section{Éditeur}

Société Internationale pour l'Histoire du Français Langue Étrangère ou Seconde

\section{Édition imprimée}

Date de publication : 1 décembre 2015

Pagination : 11-34

ISSN : 0992-7654

\section{Référence électronique}

Jean-François Zorn, "Le rôle des écoles dans la mission des Églises anglicanes et protestantes du Sud : héritages, mutations, perspectives », Documents pour l'histoire du français langue étrangère ou seconde [En ligne], 55 | 2015, mis en ligne le 01 janvier 2018, consulté le 26 mars 2023. URL : http:// journals.openedition.org/dhfles/4259; DOI : https://doi.org/10.4000/dhfles.4259

Ce document a été généré automatiquement le 26 mars 2023.

Tous droits réservés 


\title{
Le rôle des écoles dans la mission des Églises anglicanes et protestantes du Sud : héritages, mutations, perspectives
}

\author{
Jean-François Zorn
}

1 Comme les missions catholiques, les missions anglicanes et protestantes ont fondé de nombreuses écoles dans les pays dits de mission aux XIX ${ }^{\mathrm{e}}$ et $\mathrm{XX}^{\mathrm{e}}$ siècles. Au sein du protestantisme mondial le cas des missions de langue française dont il est question dans cet article est cependant particulier pour trois raisons. Premièrement, elles sont portées par trois pays ou régions d'Europe, la France, la Suisse romande et les Vallées vaudoises d'Italie, agissant au sein de la Société des Missions évangéliques de Paris (dite Mission de Paris). Deuxièmement, originaires de pays ou de régions où l'on parle français, ces missions ont débuté au début du XIX ${ }^{e}$ siècle en Afrique australe, dans une région colonisée vers la fin du siècle par la Grande-Bretagne, de sorte que les langues utilisées dans ces écoles sont l'anglais comme langue véhiculaire et les langues locales comme langues vernaculaires. Troisièmement, vers la fin du XIX ${ }^{e}$ siècle, ces missions connaissent un nouveau développement, au moment des changements d'influence voire de souveraineté dus à l'ébranlement colonial dans les pays passés sous influence française : le Gabon en Afrique subsaharienne, Madagascar dans l'océan Indien, Tahiti et Nouvelle-Calédonie dans l'océan Pacifique. Dans ces pays, les missionnaires français viennent alors remplacer les missionnaires américains et britanniques et, aux côtés des missionnaires, des instituteurs sont envoyés pour participer à la francisation de ces pays nouvellement conquis par la France.

2 La question de la langue de communication dans la mission devient alors problématique car non seulement le français est un véhicule de la colonisation, mais se pose à nouveaux frais la question du choix de la langue pour un protestantisme soucieux de traduire la parole biblique dans les langues du peuple. 
3 Dans cet article, je voudrais tout d'abord rappeler quelques éléments doctrinaux des missions protestantes concernant l'école. Ensuite je montrerai comment ces dernières ont dû s'adapter aux changements survenus à la période coloniale avec leurs conséquences sur la formation et le profil des missionnaires. Enfin j'évoquerai l'évolution plus récente de l'éducation populaire dans les Églises protestantes nées de la mission.

\section{Le temple-école dans la période précoloniale}

Dans une lettre datée de 1842, François Maeder, en poste à Beersheba au Lesotho, donne pour la première fois aux «amis» de la Mission de Paris une description de l'école qu'il dirige :

Vous trouverez ci-joint chers parents, la traduction de quelques lettres écrites en langue sessouto par des écoliers de notre grande école ; je vous envoie les originaux eux-mêmes. Je dois vous faire remarquer que c'est la première fois que ces enfants ont la plume à la main, car jusque-là nous ne pouvions les pourvoir de papier, d'encre et de plume. Soyez donc indulgents pour eux et pour moi leur maître d'école. Je ne vous envoie pas ces lettres parce qu'elles sont bien écrites, mais plutôt à cause de leur contenu, afin que vous puissiez juger vous-mêmes de la manière de penser et d'écrire de ces enfants. Je leur enseigne à écrire sur l'ardoise ; peut-être trouvez-vous que ces petits écrivains, tout novices qu'ils sont, sont assez avancés pour faire honte à plus d'un écolier paresseux d'Altenbourg $»^{1}$.

5 François Maeder n'est pas un pasteur missionnaire consacré, mais un laïc doté du statut d'aide missionnaire. Fils de maçon et maçon lui-même, Maeder a étudié le dessin à Strasbourg où il devient contremaître. C'est donc comme artisan missionnaire qu'il est engagé par la Mission de Paris qui l'emploie aussi comme instituteur. Arrivé au Lesotho en 1837, il construit sa maison, l'école et le presbytère pour le pasteur missionnaire².

Par sa précision et son enthousiasme, cette lettre dévoile le rôle que jouent et vont jouer les écoles au sein des missions protestantes. En 1842, il y a tout juste dix ans que l'évangélisation a démarré au Lesotho. Nous sommes dans la période précoloniale au cours de laquelle les nations occidentales ne se sont pas encore emparées des terres $d u$ sud ; elle durera un demi-siècle environ de 1800 à 1850 .

\section{Matrice du modèle scolaire protestant du Sud}

7 Les lettres des élèves que Maeder traduit en français sont écrites en sesotho, la langue d'enseignement qu'il a commencé à apprendre à la Maison des missions à Paris avant son départ pour l'Afrique et qu'il perfectionne sur place avant d'entrer en fonction. Cette lettre est assortie d'une gravure, la première à paraître dans le Journal des Missions évangéliques, bulletin officiel de la Mission de Paris; elle décrit la matrice du modèle scolaire protestant en mission à ses débuts.

Image 2000000900008615000077FEF4EA6A26.wmf

Journal des Missions évangéliques, 18e année, $4^{\text {me }}$ livraison, 1844.

8 Plusieurs détails de cette gravure permettent d'affirmer que la salle de classe de l'école de Beersheba ${ }^{3}$ est aussi une salle de culte. En effet, l'inscription frontispice Yehofa o Lerato signifie "Dieu est amour ». D'autres inscriptions sur les murs sont, à n'en pas douter, des versets bibliques comme dans beaucoup de temples protestants. La table de 
l'instituteur pourrait très bien être une chaire de pasteur. La disposition des élèves des petits garçons assis par terre, alignés comme s'ils étaient sur des bancs, des plus grandes filles assises, elles, sur des bancs latéraux - est aussi celle d'un culte. La présence de deux femmes qui dirigent la séance fait néanmoins penser à une école du dimanche, un culte d'enfants que les femmes peuvent célébrer alors que tel n'est pas le cas pour les cultes d'adultes. Cette disposition illustre donc la conjonction dans un même bâtiment, "le temple-école", d'une fonction pédagogique et d'une fonction cultuelle, conjonction encore symbolisée par la présence du boulier situé juste en dessous du verset biblique du frontispice. La salle ne comporte cependant pas de croix comme dans les temples consacrés au seul culte.

\section{Origines du modèle dans la Réforme et le Réveil au Nord}

9 Deux questions se posent concernant cette première école de la période précoloniale :

10 - D'où vient sa conception ? De la réforme protestante du XVI siècle qu'il n'est pas abusif de considérer comme une réforme à la foi théologique et pédagogique: les réformateurs voulurent combattre l'analphabétisme religieux pour arracher l'Église du Moyen Âge à ce qu'ils jugeaient être des erreurs théologiques en modelant, dès l'enfance, un type nouveau de croyant, un apprenant placé face à Dieu pour entendre la " voix vivante de l'Évangile » interprétée par le pasteur ; c'est la révolution théologique de la réforme. Mais les réformateurs durent aussi combattre l'analphabétisme tout court pour que ce nouveau croyant soit aussi un apprenant pouvant avoir personnellement accès non seulement aux Écritures mais à la culture à l'ère Gutenberg. Aussi, l'érection d'une chaire centrale et surtout l'installation de bancs dans les premiers temples, indiquent que ces bâtiments ont été conçus pour être des auditoires. Qu'enseignait-on de précis à ces enfants : les cinq piliers de la foi chrétienne : les Dix Commandements, le Credo, le Notre Père, les deux sacrements - baptême et cène. Dans ce but le Petit Catéchisme de Luther contenant ces rudiments, parut d'abord sous forme d'un abécédaire avant d'être un livre, grâce auquel les enfants apprenaient à lire. C'est dire que grâce à cette double révolution théologique et pédagogique, la réforme protestante allait produire dans la longue durée, une révolution culturelle considérable, d'autant plus que la langue dans laquelle ce premier enseignement fut donné était non plus le latin mais les langues nationales, l'allemand dans la zone luthérienne (Allemagne et pays nordiques), le français dans la zone calvinienne (France, Suisse et une partie de l'Europe centrale), l'anglais dans la zone anglicane (le Royaume-Uni).

11 - Comment cette conception de l'école se propage-t-elle en mission dans les pays du Sud au XIX ${ }^{\mathrm{e}}$ siècle ? Lorsqu'au début du XIX ${ }^{\mathrm{e}}$ siècle un réveil religieux gagne l'Europe post-révolutionnaire, les protestants entendent accomplir une "seconde réforme », et l'un des moyens qu'ils promeuvent en Europe et aux États-Unis d'Amérique comme dans les pays où commence la mission, est encore l'école ; l'école du dimanche d'abord, à laquelle il est fait allusion plus haut, la première étant créée en Grande-Bretagne en 1781 dans les quartiers populaires des villes industrielles. À nouveau il faut alphabétiser les enfants au moment où l'idée d'école pour tous les citoyens se fait jour un peu partout en Europe et aux États-Unis d'Amérique et dans les pays de mission. Ainsi le type d'école de Beersheba au Lesotho fait rapidement figure d'école pilote, on la nomme même «le Ban-de-la-Roche du Lesotho" par analogie avec l'expérience de 
christianisme social conduite par le pasteur Jean-Frédéric Oberlin dans les vallées vosgiennes. Enthousiasmé par ce qu'il découvre, le responsable de la Mission de Londres de passage en Afrique australe écrit en 1843: «Les élèves de l'école de Beersheba semblent aussi absorbés par leurs petits livres que le sont par les prix des fonds publics à Londres les négociants qui fréquentent la Bourse $»^{4}$. Quant au roi Moshoeshoe du Lesotho qui a accueilli et installé les missionnaires protestants français dans son pays en 1833, il déclare : « Les missionnaires de la Mission de Paris instruisent le peuple dans le christianisme et la civilisation, d'après les directions de Dieu et sans que personne ne les entrave $»^{5}$.

\section{L'école instrument de l'acculturation dans la période coloniale}

Si la période coloniale proprement dite débute dans la deuxième moitié du $\mathrm{XIX}^{\mathrm{e}}$ siècle au moment où, dans une concurrence désordonnée, les nations européennes se lancent à la conquête des pays du sud de la planète, elle est réglementée par la conférence dite " africaine de Berlin" (septembre 1884-février 1885). Cette conférence ne concerne $a$ priori que la conquête de l'Afrique subsaharienne, plus particulièrement centrale : son Acte général déclare la liberté commerciale sur les grands fleuves (Niger, Congo, etc.), la liberté de circulation des missions scientifiques, la liberté de tous les cultes (chrétiens, musulmans et traditionnels), et fixe les règles d'occupation et d'administration des territoires atteints par les nations occidentales qui deviennent des territoires conquis une fois le principe de l'Hinterland appliqué : chaque nation peut obtenir le droit d'occuper la portion de côte qu'elle revendique, mais cette occupation est assortie du devoir d'administrer l'arrière-pays'.

Ces principes vont a posteriori s'appliquer à d'autres territoires conquis que ceux de l'Afrique subsaharienne. À la veille de la Deuxième Guerre mondiale, c'est la quasitotalité des pays du Sud qui sont passés sous influence occidentale. Des transactions s'opèrent entre nations - la France et la Grande-Bretagne particulièrement, les deux principales nations impérialistes: Madagascar peut être conquis par la France contre une liberté d'action de la Grande-Bretagne en Égypte. La France est laissée libre d'agir à Tahiti et en Nouvelle-Calédonie où, dès la période pré-coloniale, elle avait manifesté ses velléités de conquête. Je cite ces exemples car ces trois pays placés sous une double influence politique britannique et religieuse protestante depuis le début du XIX siècle, passent sous influence française et catholique à la période coloniale. Ce transfert de souveraineté a une double conséquence concernant notre sujet: ces pays doivent passer de la langue anglaise à la langue française et les missions protestantes sont priées de participer à cette francisation par la langue sinon elles sont expulsées - et le seront quelquefois malgré leurs déclarations de loyauté vis-à-vis de la France...

\section{Francisation et laïcisation, les deux mamelles de l'école coloniale française}

14 Nous ne sommes donc plus du tout dans le contexte évoqué précédemment: c'est la période coloniale proprement dite, bien différente de la précédente. En effet, les Occidentaux qui n'étaient présents en Afrique pendant la période précoloniale qu'à travers des philanthropes, des explorateurs et des missionnaires, les uns et les autres 
dépourvus de visées impérialistes, sont désormais des conquérants : administrateurs, militaires et colons, comme nouveaux représentants de l'Occident. Ces gens exploitent et administrent les terres dont ils ont dépossédé les autorités traditionnelles même si elles demeurent associées sous des formes diverses de type consultatif à la gestion des colonies. Ainsi, le chef Moshoeshoe, cité plus haut, n'a-t-il plus, en 1910 qu'un titre honorifique, celui de vassal de la couronne britannique, car cette dernière, à travers ses gouverneurs, dirigent le pays, mais Moshoeshoe reste en place. Dans d'autres régions, particulièrement celles des colonies françaises, la plupart des chefs traditionnels qui n'avaient ni le statut ni la stature d'un Moshoeshoe ont disparu, soit exilés, soit pour les plus récalcitrants exécutés.

Pendant cette époque coloniale qui va durer jusqu'au moment des indépendances en 1960, l'école se développe considérablement. Non seulement elle prospère, mais elle s'autonomise au sein des missions; dans le protestantisme l'école n'est plus dans le temple, mais à côté de lui comme une œuvre essentielle de la mission à côté de l'hôpital et du centre artisanal. Le personnage de l'instituteur se distingue nettement de celui du pasteur et ses effectifs grandissent et se féminisent. Ainsi, entre 1885 et 1914, les effectifs globaux des instituteurs et institutrices missionnaires de la Mission de Paris s'élèvent à 83 sur 108 pasteurs missionnaires, contre 6 sur 65 pendant la période précoloniale (1822 à 1885) ${ }^{7}$. Laïc, l'instituteur est désormais formé à l'école normale avant son départ, il est diplômé et obtient le statut de missionnaire après avoir suivi quelques mois de formation théologique à l'École de la Mission de Paris. Ce statut officiel est exigé par le gouvernement français pour pouvoir exercer la profession d'instituteur dans les colonies et dans les Établissement français d'Outre-Mer (Tahiti) où la Mission de Paris peut ouvrir des écoles privées de formation d'instituteurs.

Les écoles protestantes, et chrétiennes en général, deviennent donc des institutions majeures de la mission pendant la période coloniale. Leurs effectifs sont souvent plus importants que les écoles du gouvernement colonial, tout simplement à cause de leur antériorité et de leur enracinement. Dans les colonies françaises l'école est l'instrument majeur de la colonisation par la francisation et la laïcisation, procédant ainsi à une acculturation de la population autochtone : la langue française devient l'instrument du formatage des esprits à la civilisation occidentale. Le modèle français qui sépare l'enseignement des sciences et des lettres de l'enseignement religieux cherche à s'imposer dans les colonies, mais les missions résistent à cette conception, ce qui provoque des conflits singuliers comme à Madagascar connu comme un champ d'expérimentation du modèle scolaire français dans une société longtemps dominée par le modèle anglais ${ }^{8}$.

17 Il convient à cet endroit, de rappeler que l'un des théoriciens de la colonisation française n'est autre que Jules Ferry, par ailleurs artisan de l'école républicaine laïque en France... Ce sont d'ailleurs les mêmes lois Ferry des années 1880 qui interdisent, par exemple, aux petits Gascons de France et aux petits Fang du Gabon de parler leur langue maternelle dans la cour de l'école. Le français devient la langue obligatoire pour tous. On peut dire qu'en France, l'école publique laïque procède également d'une sorte de colonisation intérieure du pays en vue de former le creuset de la nation autour de l'unique langue française et des valeurs civilisationnelles de la république qui sont encore loin d'être partagées par tous les citoyens français à la fin du XIX ${ }^{\mathrm{e}}$ siècle.

18 Face à cette situation nouvelle, créée par la colonisation, quelle est la position des missions protestantes pendant la période coloniale? Pour essayer de circonscrire la 
réponse à cette question, je vais la traiter dans ses grandes lignes telle qu'elle émerge dans les conférences missionnaires internationales anglicanes et protestantes, depuis celle d'Édimbourg en 1910, jusqu'au moment des indépendances.

\section{Mission, éducation et christianisation à la conférence missionnaire mondiale d'Édimbourg en 1910}

19 La conférence d'Édimbourg traite toutes les grandes questions qui se posent à la mission alors que l'optimisme et le triomphe de la civilisation occidentale n'ont pas encore été touchés au cœur par le premier conflit mondial de 1914. Parmi les huit thèmes traités par la conférence l'éducation tient une bonne place avec comme titre: «L'éducation en relation avec la christianisation de la vie nationale». Cette formulation (traduite de l'anglais, langue officielle de la conférence) est à plus d'un titre intéressante : elle énonce deux objectifs majeurs assignés à l'éducation dans le cadre de la mission : 1) «christianiser » est une action plus large qu'évangéliser; elle signifie que l'éducation est une action culturelle globale visant à diffuser et infuser le christianisme dans la société. On est donc loin du processus de laïcisation mis en œuvre en France à la même époque. 2) Christianiser «la vie nationale " est cet objectif qui cible un ensemble homogène désigné par le terme "nation ", alors que n'existent encore que des empires; cet objectif tente de circonscrire un mouvement de type nationaliste indigène qui se dessine afin de le canaliser, dans le cadre colonial, vers la création de futurs États-nations peuplés de citoyens éduqués et pourvus d'élites autochtones pour diriger ces États le moment venu.

L'école est donc aux avant-postes de ce vaste projet. En témoigne le questionnaire qui fut envoyé deux ans avant la tenue de la conférence à toutes les missions anglicanes et protestantes dans le monde qui étaient à la tête, selon les statistiques de l'époque, de 31462 établissements scolaires de niveau primaire essentiellement, mais déjà, dans certains pays d'Asie, de niveau secondaire et supérieur, le tout regroupant 1361500 élèves dans le monde, chiffres d'ailleurs sensiblement équivalents à ceux des missions catholiques $^{10}$. Ce questionnaire comptait quatorze questions. Je ne peux toutes les présenter, je vais les regrouper par thèmes, et surtout indiquer les orientations que les trois cents réponses ont tracées et qui ont été regroupées dans un volume de 471 pages $^{11}$ !

Un premier thème porte sur les objectifs de l'œuvre scolaire ; la $1^{\text {ère }}$ question demande si « l'école est faite pour les enfants des chrétiens ou pour les non-chrétiens ", et la $2^{\mathrm{e}} \mathrm{si}$ "l'enseignement chrétien a formé des hommes et des femmes à la fois chrétiens dans leurs convictions et indigènes dans leur pensée, leur vie affective, et leur regard sur la vie » et «si cet enseignement a globalement provoqué un style de vie meilleur au sein de la population non chrétienne »; la $4^{\mathrm{e}}$ question s'intéresse aux rapports entre la politique scolaire des missions et celle des autorités civiles, en soulevant notamment le problème difficile de la "clause de conscience ", qui a trait à l'assiduité obligatoire ou facultative des élèves non chrétiens aux cours de religion et aux exercices religieux chrétiens.

22 Un deuxième thème tente de préciser l'interaction entre l'éducation donnée par les missions et la société indigène; ainsi la $3^{\mathrm{e}}$ question se demande : "quelle influence le nationalisme local a-t-il sur la politique scolaire des missions?", alors que la $5^{\mathrm{e}}$ question demande que soient précisées «les relations établies ou à établir entre les 
matières et les méthodes de l'enseignement chrétien et la culture indigène, ses modes de pensée, les traditions, la littérature "; tout naturellement la $6^{\mathrm{e}}$ question s'interroge sur « la langue de l'enseignement : langue locale vernaculaire, langue nationale du pays si elle existe ou encore langue européenne ».

Un troisième thème porte sur les populations visées par l'école des missions, notamment les filles, délaissées par les pouvoirs coloniaux $\left(7^{e}\right)$ et les débouchés qui leur sont offerts; les futurs travailleurs manuels à travers l'enseignement professionnel agricole et industriel, types d'enseignement privilégié par les pouvoirs coloniaux $\left(8^{\mathrm{e}}\right)$.

Un quatrième thème porte sur des questions pédagogiques : le matériel pédagogique $\left(11^{\mathrm{e}}\right)$, la formation des maîtres qui implique la collaboration des diverses missions pour ouvrir des écoles normales, organiser l'inspection des écoles, imaginer des écoles spécialisées en médecine, créer des foyers et des internats, projeter la création d'établissements d'enseignement supérieur $\left(12^{\mathrm{e}}, 13^{\mathrm{e}}\right)$, les finances $\left(14^{\mathrm{e}}\right)$, le suivi des anciens élèves $\left(9^{\mathrm{e}}\right)$.

Les réponses reçues sont regroupées en 1909, résumées et évaluées dans un gros rapport préparatoire mis à la disposition des délégués avant la conférence de $1910^{12}$. Quatre points forts sont mis en relief ${ }^{13}$.

27 - «En premier lieu, les missions protestantes sont unanimes pour affirmer la nécessité et la valeur de l'école dans l'éventail des activités missionnaires. Il n'existe donc plus guère d'objection contre l'engagement des missions et des Églises dans le domaine éducatif ».

28 - «En second lieu [...] le christianisme doit devenir partout indigène et ne plus apparaître comme un corps étranger [...], car l'école prépare les hommes et les femmes qui devront penser le christianisme dans leur perspective nationale propre [...]. Partout les nationalismes montent. On entend de nouveaux slogans: la Chine aux Chinois, l'Afrique aux Africains! Malgré tout, les écoles chrétiennes sont appréciées et recherchées: elles peuvent souvent atténuer les préventions et l'hostilité contre les missions étrangères ».

29 - «En troisième lieu [...] l'enseignement ne peut être un simple transfert de connaissances. L'éducation proposée par les missions protestantes est envisagée comme une formation humaine totale, qui inclut l'éducation morale et religieuse [...]. Ainsi les missions protestent contre le laïcisme qui exclut tous les sujets religieux des programmes des écoles publiques ».

30 - «En quatrième lieu, un certain consensus s'est fait jour sur une puissante motivation de l'engagement des missions dans l'œuvre scolaire, c'est le sentiment du devoir de solidarité et d'entraide envers les nations démunies, qu'elles soient chrétiennes ou non chrétiennes [...] Dans cette optique, l'école confessionnelle n'est plus tellement considérée comme un moyen d'évangélisation destiné à faire des chrétiens à longue ou à brève échéance, mais comme une simple action humanitaire ».

31 Deux tensions propres à l'éducation scolaire chrétienne sont bien identifiées à Édimbourg, l'une autour de la sécularisation, l'autre autour de l'inculturation :

32 - en 1910, la séparation de l'école et de l'Église, évolution déterminante du processus de sécularisation partout à l'œuvre dans le monde, en France particulièrement avec la laïcisation, n'est pas consommée, mais elle est en marche. À Édimbourg, on a dit à la fois que mission et éducation vont de pair parce qu'on pense encore possible de christianiser la société tout entière, mais aussi que l'éducation chrétienne a d'abord un 
objectif humanitaire avant celui d'évangélisation. Ceci ouvre la porte à une éducation et une école conduites par les missions puis les Églises sans but prosélyte explicite ;

- à Édimbourg, on dit que l'école doit être un agent déterminant d'une inculturation globale, à travers le christianisme qui ne doit pas apparaitre comme une religion étrangère, les langues locales qui ne doivent pas disparaître et plus généralement à travers tout ce qui contribue à la reconnaissance de ce qu'on nomme alors «le génie propre des indigènes "; mais on dit aussi que l'école va provoquer une acculturation des enseignés et en les ouvrant à d'autres valeurs, celles de la civilisation qu'on nomme occidentale parce qu'elle domine encore le monde et qu'elle est porteuse des valeurs de la liberté, des sciences et des techniques. Les langues étrangères ont évidemment leur rôle à jouer dans cette acculturation.

Ces deux tensions vont traverser toute la période coloniale comme j'ai pu le constater à travers mes propres recherches. Avant de passer à la période suivante, l'époque postcoloniale, je veux juste citer une phrase recueillie dans une brochure éditée par la Mission de Paris en 1957 intitulée Regards sur nos champs de mission alors que le processus d'autonomie des Églises était enclenché et que l'indépendance politique des pays du Sud se profilait: "La tâche change de forme, se complique, devient plus difficile, mais elle reste la même : évangélisation des régions païennes, édification de l'Église, formation des ouvriers, direction des grandes écoles et de la politique scolaire, œuvres de jeunesse, action sociale $»^{14}$.

\section{Éducation et développement : permanences et ruptures à l'époque postcoloniale}

Je fais débuter cette période dite postcoloniale aux années 1960, période de l'indépendance de la plupart des pays du Sud. Les écoles sont désormais passées sous la responsabilité des Églises anglicanes et protestantes qui créent des directions de l'enseignement confessionnel dans chaque pays à côté des écoles publiques, celles-ci n'assurant souvent que la moitié à peine de la totalité de l'enseignement national. Mais les subsides pour les écoles venus des anciennes Sociétés de mission connaissent une diminution ou une modification, car ces sociétés disparaissent ou se transforment en services missionnaires des Églises en utilisant d'ailleurs la nouvelle terminologie des États : on ne parle plus de colonisation mais de coopération. Dans ce nouveau cadre institutionnel, le budget missionnaire des écoles des Églises pour les pays du Sud, passe sous la responsabilité de ces services qui vont tenter d'assumer l'héritage des œuvres scolaires et accompagner leur évolution. En France, le Service protestant de mission, le Défap va, comme la Délégation catholique à la Coopération pouvoir envoyer des Volontaires du Service national (VSN) de 1963 jusqu'à la fin de la conscription en 2002, particulièrement dans les collèges et lycées dirigés par les Églises. Dans ces établissements, l'enseignement est donné exclusivement en langue française, les langues vernaculaires, nationales quand il y en a une comme c'est le cas à Madagascar, sont réservées à l'enseignement primaire.

\section{« L'éclipse du scolaire » dans la mission protestante}

36 Je voudrais repartir de mon expérience personnelle de VSN et de mes propres recherches archivistiques, pour essayer d'esquisser les caractéristiques de la période 
qui commence où « les œuvres scolaires » deviennent un problème, alors que jusque-là elles paraissaient une solution. Le terme "œuvre" a un sens précis dans le protestantisme. Il confirme à la fois l'engagement pratique des Sociétés de mission puis des Églises dans des actions diverses, scolaires, mais aussi sanitaires, l'école et l'hôpital qui ont marqué d'un certain prestige la mission dans la période coloniale.

Certes, « sans les œuvres, la foi est morte ", tous les protestants se reconnaissent dans cette formule biblique souvent reprise (épître de Jacques, chap. 2). Mais on se demande si c'est vraiment aux Églises d'assumer l'éducation, la formation et l'enseignement des citoyens, mais pas plutôt aux États. De ce côté-là les protestants français favorables à la laïcisation de l'enseignement en France à la fin du XIX siècle le sont également en mission. Dans la vision protestante des choses les œuvres scolaires sont donc secondes, pour ne pas dire secondaires car, de plus, on ne croit pas au salut par les œuvres. Je ne voudrais pas majorer le poids de cette croyance, ni en faire une clef de lecture de la récente histoire des écoles et des œuvres protestantes dans les pays du Sud; mais je crois qu'elle permet au moins d'introduire, sinon d'expliquer, ce que l'on constatait déjà pendant la période coloniale autour de la Deuxième Guerre mondiale, puis au moment des indépendances, à savoir une crise de l'école qui se traduit par « une éclipse du scolaire " à compter des années 1930 pour reprendre l'expression imagée du professeur Marc Spindler qui a analysé ce phénomène à travers les conférences missionnaires internationales qui se tiennent tous les dix ans depuis celle d'Édimbourg en $1910^{15}$.

Je ne peux pas reprendre toute la démonstration de Spindler, mais il dit en substance que les conférences qui ont suivi celle d'Édimbourg ont toutes poursuivi l'un des objectifs esquissés en 1910, c'est-à-dire adapter l'école dont le modèle venait de l'Occident aux réalités locales des pays de mission. Ainsi, on invente dans l'Entre-deuxguerres le concept d'« école de brousse » pour mettre en avant l'éducation populaire rurale en contrepoint d'une éducation élitaire urbaine; puis on ajoute le concept d'« éducation religieuse » à un moment où des idéologies laïcistes et communisantes, d'un côté, fascistes et autoritaires de l'autre, se répandent dans le monde en décriant le christianisme, l'une parce qu'il serait contraire à la liberté et à la science, l'autre, à l'inverse, parce qu'il serait un apprentissage de la liberté conduisant au désordre et à l'insoumission.

39 Ces deux influences - le communisme et le fascisme - on le comprend aisément, constituent une menace pour les Églises qui craignent alors que les élèves de plus en plus éduqués et influencés par ces idéologies, ne désertent la vie religieuse et paroissiale. Que fallait-il faire se demande alors la conférence missionnaire mondiale de Madras en 1938 au vu des moyens, forcément limités, des Sociétés de mission et des Églises naissantes? Continuer une politique scolaire avec ses instruments traditionnels ou élargir la mission éducative de l'Église avec des moyens pédagogiques nouveaux en développant par exemple, la littérature, le cinéma, le théâtre, etc., à travers des mouvements de jeunes et d'étudiants? Ces questions restées en suspens avant la Deuxième Guerre mondiale ne trouvent leur réponse qu'après-guerre, mais se traduisent bel et bien par la fameuse « éclipse du scolaire ».

Entendons-nous bien sur le sens de la formule : elle ne signifie pas que les écoles et tous les autres établissements scolaires disparaissent, quoique la débâcle après 1947, des missions en Chine par exemple, le «fleuron de l'œuvre scolaire et universitaire des 
missions chrétiennes ", fait couler en deux ans près de la moitié de l'œuvre scolaire protestante mondiale...

41 Mais cette éclipse du scolaire montre que l'école n'occupe plus dans les réflexions des conférences missionnaires de l'Entre-deux-guerres la place qu'elle occupait à Édimbourg. Les statistiques, par exemple, disparaissent des rapports dès la conférence missionnaire mondiale de 1938. Pourquoi ? Spindler donne trois raisons : la première, théologique, se rapproche de ce que je disais plus haut du regard protestant sur les œuvres : elles ne sont pas équipées pour porter la préoccupation centrale de la mission, c'est-à-dire l'évangélisation, ce n'est pas leur spécificité, cela doit revenir aux Églises locales auxquelles il faut donner les moyens de cette mission. Comme, au même moment, les écoles sont progressivement dévolues à ces Églises qui cherchent à grandir et à s'imposer, elles donnent moins de moyens à leurs écoles, c'est la deuxième raison de l'éclipse du scolaire. À cela il faut ajouter une troisième raison: au moment de la décolonisation, des critiques de la part des intellectuels du Sud relayées par le mouvement tiers-mondiste puis les études postcoloniales au Nord tombent sur l'école $\mathrm{du}$ temps colonial et missionnaire. On connaît la formule assassine qui dit qu'on a enseigné aux Africains et aux Océaniens que leurs ancêtres étaient les Gaulois! Quoi qu'il en soit, la part incontestable d'acculturation provoquée par l'école fait plus ou moins bon ménage avec ce qu'elle a apporté comme moyens de compréhension de soi. Le contexte colonial a déformé ces objectifs en les radicalisant: l'acculturation s'est mutée en déculturation, la compréhension de soi en révolte et quelquefois en négation de soi, la langue du colon comme instrument de connaissance du monde est vue comme la langue de l'aliénation culturelle, etc. L'école née au temps colonial et missionnaire paraît ainsi, pendant les quelques décennies qui précèdent et suivent les indépendances, comme anesthésiée par la critique postcoloniale et postmissionnaire.

\section{Comment retrouver la mission éducative de l'Église?}

Bien que répandues dans les milieux d'églises, cette critique ne les conduit pas pour autant à renoncer à leurs écoles. Pour le vérifier, trois ans après la disparition de la Mission de Paris, l'organisme international qui lui succède, une Communauté internationale d'Églises en mission adresse à toutes ses Églises membres un questionnaire visant à préciser quels sont, selon elles, le rôle et le devenir de leurs écoles ${ }^{16}$. À la question de savoir si l'Église se reconnaît une mission éducative, plusieurs réponses affirment que la mission de l'Église est «d'aider à prendre conscience de ce qu'est l'homme devant Dieu [...] un être adulte sur tous les plans dont on aura développé l'esprit critique et constructif, son sens de la liberté et de la responsabilité ", mais que l'école n'est qu'un des vecteurs parmi d'autres - l'enseignement religieux, l'animation de la jeunesse, l'organisation de l'Église - de cette mission éducative. Une liste des difficultés des écoles protestantes est dressée; elles portent essentiellement sur leur identité confessionnelle, pas sur leur projet éducatif: on déplore essentiellement le manque de motivations des parents pour envoyer leurs enfants à l'école protestante, le détachement du corps enseignant de l'Église, celui-ci ne souhaitant quelquefois pas le maintien du caractère confessionnel des écoles et des institutions et se montrant même parfois hostile à la présence d'un enseignement religieux à l'école, la rareté des vocations pour un enseignement et un témoignage chrétiens. On déplore en outre les traitements trop faibles des enseignants qui obligent 
à un recrutement de gens peu qualifiés, le manque de moyens financiers, la dépendance extérieure des écoles confessionnelles.

43 À côté de l'enseignement public et face à la velléité de certains États (Bénin, Congo, Madagascar) de nationaliser tout l'enseignement au cours des années 1970, toutes les réponses affirment que "jusqu'à nouvel ordre ", les Églises du Sud ont le devoir de maintenir des écoles confessionnelles pour deux types de raisons, les unes tenant à l'insuffisance quantitative et qualitative des écoles publiques, les autres à ce qui a fait et fait encore la spécificité de l'enseignement confessionnel: à la fois répondre aux besoins même de l'Évangile et être l'aiguillon de l'État pour que ne soient oubliés ni le bien du pays, ni la recherche pédagogique tournée vers le développement, ni l'adaptation de l'enseignement aux nouveaux besoins de la société. Telle est donc la position des Églises membres de cette Communauté internationale d'Églises qui succède à la Mission de Paris en 1971.

\section{Vers une Église sentinelle et une école de la libération}

$44 \mathrm{Au}$ fond, à travers leurs écoles, mais pas seulement elles, les Églises anglicanes et protestantes des pays du Sud de la période postcoloniale et postmissionnaire se perçoivent des sentinelles de l'éducation du peuple. Mais devant cette prétention, Samuel Aklé, alors directeur de l'enseignement protestant du Bénin, fait en 1974 le commentaire suivant au vu des résultats de l'enquête citée plus haut : "Je me permets de faire remarquer que les Églises tiennent beaucoup à leur témoignage dans le domaine de l'enseignement et surtout de l'éducation. Aussi doivent-elles arrêter des œuvres de prestige pour s'orienter de plus en plus vers des réalisations innovatrices. Elles sont si souvent complices des systèmes scolaires économiques, donc politiques en place, qu'elles perdent malheureusement de vue leur rôle prophétique (souligné dans le texte). L'évangélisation, qui est la mission primordiale de l'Église, devra renouveler sa vision du monde, son analyse des signes des temps, adapter ses méthodes, rejeter le conformisme, ne pas craindre de témoigner pour former des hommes nouveaux ${ }^{17}$.

Ce commentaire fait mouche et la Communauté des Églises multiplie les initiatives pour engager les églises à évaluer leurs œuvres. En août 1974 un colloque inter-Églises d'Afrique-Madagascar-Pacifique de l'Enseignement protestant se tient à Douala au Cameroun sur le thème «L'engagement prophétique de l'Église dans l'éducation » au cours duquel l'accent est mis sur des actions concrètes visant à bien définir ce qu'on entend par engagement prophétique et par éducation ${ }^{18}$. Un des participants lance l'idée que l'école chrétienne doit être une « école-projet » et non une « école-reflet ».

Pour illustrer comment cette intuition s'est concrétisée dans le domaine éducatif, je voudrais citer l'un de ces réseaux régionaux africains qui a vu le jour en mars 1989 : le Réseau École et Développement (RED) créé à l'initiative du directeur de l'enseignement protestant de Côte d'Ivoire, le pédagogue Jean-Djoman Agbassi. C'est un mouvement de rénovation de l'école sur la base d'une pédagogie de la libération. Le RED a tôt fait le constat d'une insatisfaction dans l'accomplissement de la mission éducative que s'étaient assignée de longue date les Églises d'Afrique du fait de l'augmentation de la pauvreté et du chômage massif des jeunes pourtant formés et diplômés. Ainsi l'objectif global du RED consiste à " articuler l'école sur son environnement local, national et international pour penser globalement et agir localement; à faire de l'école un instrument de développement durable à travers l'agriculture, l'art, l'artisanat, la 
culture, la démocratie, l'écologie, les droits sociaux, l'éducation scientifique et interculturelle, à stimuler la contribution active de l'élève aux processus d'acquisition de connaissances $»^{19}$.

Pour diffuser ses idées et soutenir son action, un bulletin de liaison trimestriel RED/ ACTION est lancé, des ouvrages sont publiés comme Correspondances, vers une pédagogie de la libération et plusieurs autres encore ${ }^{20}$. Des colloques sont organisés au Togo en 1994, au Cameroun en 1996. En 1994 est lancé le concept d'écoles pilotes appelées à être créées au sein des établissements scolaires traditionnels pour tenter d'insuffler aux enseignants, aux enfants et aux parents d'élèves l'esprit et les méthodes des écoles pilotes. En 2007 un bilan de l'action est dressé : 139 écoles pilotes ont été créées en Afrique de l'ouest et centrale qui touchent 44060 élèves dans 834 classes dans lesquelles on compte $40 \%$ de filles. 960 enseignants sont concernés. Dans ce bilan, modeste mais encourageant, sont tracées les perspectives pour la période 2007-2010 intitulées «Enracinement de la pédagogie de la libération dans les Églises membres du RED ». C'est dire qu'il reste encore beaucoup de travail à faire, rendu difficile aujourd'hui par les difficultés surgies en Côte d'Ivoire d'où est partie l'initiative et où se trouvent encore les cerveaux du RED. À l'heure qu'il est le RED ne s'est pas encore relevé de la guerre en Côte d'Ivoire et pour préparer cet article, je ne suis pas parvenu à rétablir le contact avec ses responsables.

\section{Conclusion : rôle de la langue française dans le type d'école de la libération}

48 En conclusion, je ferai trois remarques concernant le rôle de la langue française dans le type d'école de la libération dans les pays du Sud.

49 - La première repart de cette expérience du Réseau École et Développement né en Côte d'Ivoire. Il est bien évident qu'un tel réseau africain, du fait de son caractère transfrontalier, ne peut qu'utiliser la langue française pour communiquer entre les directions d'enseignement et les réseaux d'enseignants et ceux de formateurs des différents pays. Il n'est pas exclu que des manuels de formation puissent être, ici ou là, traduits en langues locales pour être mieux utilisés à la base, mais à ma connaissance, je n'ai jamais vu de telles traductions dans ce Réseau. Même si c'est un truisme, il paraît donc évident que comme vecteur de la problématique d'une école de la libération, la langue française est elle-même un élément constitutif de la libération recherchée. Mais c'est difficile à dire, encore plus à écrire, tant la langue française a été associée à l'entreprise de colonisation par la francisation, arme essentielle de l'idéologie coloniale des $\mathrm{XIX}^{\mathrm{e}}$ et $\mathrm{XX}^{\mathrm{e}}$ siècles. Il faut encore du temps, beaucoup de temps, pour que cette association de la langue française et de la libération puisse être admise. C'est un changement de mentalité qui doit s'opérer. Seuls les ressortissants des pays du Sud peuvent l'assumer avec notre soutien pas seulement sentimental mais logistique, en équipant l'entreprise de libération de moyens pédagogiques appropriés.

- La deuxième remarque concerne l'habitus culturel des missionnaires protestants visà-vis des langues à enseigner dans les pays du Sud. On aura compris à partir de cet article que les missions anglicanes et protes-tantes ont traditionnellement enseigné en langue vernaculaire, tant dans les Églises que dans les écoles. Ce n'est qu'à la période coloniale qu'elles ont été obligées de passer au français, ce fut d'autant plus rude que ce 
passage s'est souvent fait, je l'ai à peine évoqué, au moment où des missionnaires protestants non français étaient chassés des pays passant sous domination française à l'heure de la conquête coloniale au Gabon, Madagascar, Polynésie, et NouvelleCalédonie ou, après la Première Guerre mondiale, au Cameroun et au Togo. Comme de surcroît une concurrence s'est engagée alors entre les missions catholiques souvent arrivées avec les Français et les missions protestantes venues avec les Britanniques et les Américains, politique et religion se sont mélangées ne permettant pas de clarifier, dans ce contexte, le rôle émancipateur de l'école pourtant régulièrement prôné par les missionnaires.

51 - Enfin, troisième et dernière remarque, je reviens de Madagascar où j'ai assumé une mission d'enseignement d'une dizaine de jours dans une Faculté de théologie protestante censée être de niveau universitaire à Fianarantsoa. Mon enseignement était donné en français et mes cours étaient distribués imprimés. Malgré ces facilités, une traduction simultanée en malgache a été jugée nécessaire, tant le niveau de compréhension du français des étudiants, pourtant tous bacheliers, était faible. Cette situation est le résultat d'une politique de malgachisation de la langue à tout niveau d'enseignement pendant vingt ans de 1972 à 1992. Ainsi la jeunesse de ce pays, relativement avancée dans la maîtrise de la langue française a-t-elle désappris le français tout en apprenant mal le malgache par manque de moyens. Depuis cette période difficile, le pays est revenu en arrière, redonnant tantôt au français le statut de seconde langue officielle tantôt de langue étrangère. Mais un collègue universitaire linguiste me faisait remarquer que face à ce désapprentissage des deux langues, Madagascar était en train de retourner à l'oralité des échanges, les écrits ne pouvant plus guère servir à les médiatiser. Aussi, Madagascar, comme pas mal d'autres pays du Sud, doit-il accomplir une révolution de type Gutenberg et sur ce point, ce même collègue me disait que le passage par la langue française était probablement indispensable. À bon entendeur salut !

\section{NOTES}

1. Lettres de François Maeder, missionnaire de la station de Béerséba au Lessouto, Journal des Missions évangéliques, 1844, p. 134.

2. Cf. Théophile Jousse, «François Maeder» (article nécrologique), dans Journal des Missions évangéliques, 1888, p. 211.

3. Selon l'orthographe actuelle.

4. «Détails sur l'école de Béerséba donnés à la ville du Cap par le docteur Philip (surintendant de la Mission de Londres) », Journal des Missions évangéliques, 1844, p. 225.

5. Règlements du gouvernement de la ville de Béerséba du 22 janvier 1844. Microfilm, archives de la Mission de de Paris.

6. Sur la conférence africaine de Berlin et ses résultats on consultera Henri Brunschwig, Le Partage de l'Afrique noire, Paris, Flammarion, « Champs d'histoire », 20092. 
7. Jean-François Zorn, «L'École de la Société des Missions 1822-1971 ", dans Jacques Gadille et Marc Spindler, Sciences de la mission et formation missionnaire au XXe siècle, Actes de la XIIe session du CREDIC, Lyon/Bologna, LUGD/EMI, 1992, p. 294.

8. Cf. Jean-François Zorn, « La séparation des Églises et de l'État à Madagascar », dans Jean-Pierre Chantin et Daniel Moulinet (dir.), La Séparation de 1905. Les hommes et les lieux, Paris, Éditions de l'Atelier, 2005, p. 201-217.

9. À l'époque, il pouvait à la fois recouvrir la notion biblique « les nations », c'est-à-dire les pays dit païens et la notion politique profane occidentale d'« État-nation » démocratique.

10. Ces statistiques sont extraites de Statistical Atlas of Christian Missions: Containing a Directory of Missionary Societies, A Classified Summary of Statistics, An Index of Mission Stations, and a Series of Specially Prepared Maps of Mission Fields, 1910, p. 63-85.

11. World Missionary Conference, 1910, Report of Commission III, Education in Relation to the Christianisation of National Life, World Missionary Conference, 1910, Edinburgh and London/ New York, Chicago, and Toronto, Oliphant, Anderson, \& Ferrier/ Fleming H. Revell Company, 1910. Cf. sur le sujet : Marc Sindler, «Les nations à l'école du Christ : bilan de la Commission III à Édimbourg ", dans La Conférence missionnaire mondiale, Édimbourg 1910, dossier dirigé par Jean-François Zorn, Claire-Lise Lombard, Jacques Matthey, dans Histoire \& Missions Chrétiennes, $\mathrm{n}^{\circ} 13$, mars 2010, p. 33-45.

12. Jean de Visme, "Les Missions et l'instruction", dans Journal des Missions évangéliques, novembre 1910, p. 368-381.

13. Je cite ici des extraits du compte rendu qu'en donne Marc Spindler, art. cit., p. 40-41.

14. Regards sur nos champs de mission, SMEP, Paris, 1957, Fiche « Regards sur Madagascar».

15. Marc Spindler, «La politique scolaire des Missions protestantes à travers les conférences du Conseil international des missions (1910-1961) », dans Écoles et missions chrétiennes, Actes de la VIIIe session du CREDIC, Salamanque (24-27 août 1987), Lyon, Université Jean Moulin et Facultés catholiques, 1988, p. 108-125.

16. Cf. le dépouillement de cette enquête : Samuel Aklé, « Églises et écoles protestantes d'outremer ", dans Foi et Éducation, janvier-mars 1977, nº 17, p. 2-8.

17. Ibidem, p. 8.

18. Procès-verbal du colloque "Afrique-Madagascar-Pacifique » de l'enseignement protestant tenu au collège Alfred Saker (Douala) du 22 au 27 août 1974 sur le thème L'engagement prophétique de l'Église dans l'éducation, document dactylographié, Archives Défap.

19. Cf. site Internet : <http://recodev.com/index.php>

20. Yaoundé, Clé, 1999. Cf. également, Guide de l'aumônier, Abidjan, RED, 2002 ; Paix : Avançons !, Abidjan, RED, 2004 ; Contre le Sida, RED/CEDA, Abidjan, 2004.

\section{RÉSUMÉS}

L'école a joué un rôle fondamental dans les Missions anglicanes et protestantes des pays du Sud. $\mathrm{Si}$, au début du XIXe siècle, la salle de classe est la même que celle du temple rappelant l'aspect pédagogique de la réforme protestante, à la fin de ce siècle l'école n'est plus dans le temple, mais à côté de lui tout en demeurant une œuvre essentielle de la mission. La période coloniale proprement dite commence, et là où la mission et son école ont précédé la colonisation française, des tensions surgissent entre le colon qui veut franciser et laïciser et l'instituteur missionnaire 
qui enseigne en langue locale et ne perd pas de vue l'objectif religieux. Mais, dès le début du XXe siècle une séparation s'opère entre les Missions et leurs écoles visant à écarter le prosélytisme de ces dernières et les ouvrir à tous. L'école doit cependant demeurer un agent d'inculturation et non d'acculturation pour rester " indigène ", ne pas apparaitre " étrangère " au contexte dans lequel elle se développe tout en s'ouvrant aux valeurs universelles. Avec la période postcoloniale qui débute lors de l'indépendance des principaux pays du Sud et de l'autonomie des Églises anglicanes et protestantes, ces dernières créent des directions privées d'enseignement primaire et secondaire. L'émergence de l'enseignement public provoque une éclipse de l'enseignement protestant traditionnel qui se tourne alors vers l'éducation populaire et communautaire, les Églises se présentant elles-mêmes comme les sentinelles d'une formation dite de la libération.

\section{INDEX}

Keywords : protestants, missionaries, colonies, schools, languages

Mots-clés : protestants, missionnaires, colonies, écoles, langues

\section{AUTEUR}

\section{JEAN-FRANÇOIS ZORN}

Institut Protestant de Théologie de Montpellier -

Université Paul-Valéry de Montpellier 